\title{
Reconstrução de curvas paramétricas por meio de uma abordagem probabilística
}

\author{
Reconstruction of parametric curves through a probabilistic approach
}

Marcones de Oliveira Silva
Universidade Federal de Alagoas (UFAL), Curso de Bacharelado em Matemática, Maceió, AL, Brasil
(ID) https://orcid.org/0000-0003-4445-5210, $\begin{array}{r}\text { marconesoliveira1@gmail.com } \\ \text { Thiago Amaral Melo Lima }\end{array}$
Universidade Estadual do Piauí (UESPI), Mestrado Profissional em Matemática em Rede Nacional
(PROFMAT), Teresina, PI, Brasil
(ID) https://orcid.org/0000-0003-2574-1297, thiagoamaral.mat@gmail.com

\section{Informações do Artigo}

\section{Como citar este artigo}

SILVA, Marcones de Oliveira; LIMA, Thiago Amaral Melo. Reconstrução de curvas paramétricas por meio de uma abordagem probabilística. REMAT: Revista Eletrônica da Matemática, Bento Gonçalves, RS, v. 7, n. 1, p. e3008, 15 mar. 2021. DOI: https://doi.org/10. 35819/remat2021v7i1id4262

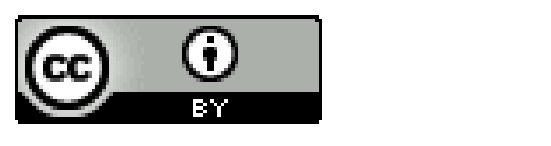

Histórico do Artigo

Submissão: 12 de junho de 2020.

Aceite: 6 de janeiro de 2021.

\section{Palavras-chave}

Curva Paramétrica

Função de Densidade de Probabilidade

Variável Aleatória

\section{Resumo}

Este trabalho trata da reconstrução de curvas paramétricas utilizando funções de densidade de probabilidade para gerar uma amostragem de pontos no domínio da curva e aproximado-a em um intervalo específico. Existem várias abordagens para amostrar curvas paramétricas, que permitem que a mesma esteja de acordo com a curvatura ou comprimento de arco. Em geral, estas técnicas estão baseadas em heurísticas, e não conseguem dar soluções ótimas. Neste artigo, pretendemos usar uma abordagem probabilística, de modo que a amostragem de pontos resultante esteja de acordo com alguma função de densidade definida no domínio da curva, colocando mais pontos onde esta densidade é maior. Por ser mais geral, esta abordagem inclui os casos mencionados acima como casos particulares. Em geral, aproximar curvas planas com base na Distribuição Uniforme se revelou mais eficiente do que tomando como referência a Distribuição Exponencial. O valor de $\lambda$ na Distribuição Exponencial interfere na aproximação de algumas curvas, sendo necessário encontrar um valor de $\lambda$ adequado para chegar a uma boa aproximação da curva. Aproximar a curva com base na sua curvatura é um método usado quando se deseja gerar mais amostras onde a curvatura é maior. Porém, esse método só pode ser usado em casos especiais uma vez que a integral da função curvatura, na maioria das vezes, é muito difícil de ser calculada. 


\section{Keywords}

Parametric Curve

Probability Density Function

Random Variable

\begin{abstract}
This work deals with the reconstruction of parametric curves using probability density functions to generate a sampling of points in the curve domain and approximate it over a specific interval. There are several approaches for sampling parametric curves, which allow it to be in accordance with the curvature or arc length. In general, these techniques are based on heuristics, and fail to provide optimal solutions. In this article, we intend to use a probabilistic approach, in the way that the resulting point sampling is in accordance with some density function defined in the curve domain, placing more points where this density is greater. As it is more general, this approach includes the cases mentioned above as particular cases. In general, approximating flat curves based on the Uniform Distribution proved to be more efficient than taking the Exponential Distribution as a reference. The value of $\lambda$ in the Exponential Distribution interferes in the approximation of some curves, being necessary to find a value of $\lambda$ suitable to get a good approximation of the curve. Approaching the curve based on its curvature is a method used when it is wanted to generate more samples where the curvature is greater. However, this method can only be used in special cases since the integral of the curvature function, in most cases, is very difficult to be calculated.
\end{abstract}

\section{Introdução}

Este artigo aborda o problema de amostragem e reconstrução de curvas paramétricas. Embora existam soluções triviais para este problema, estas geralmente não são satisfatórias por amostrarem a curva de forma não uniforme, pois são baseadas em amostragem uniforme do domínio.

As curvas paramétricas aparecem frequentemente em modelagem geométrica, em aplicações da Computação Gráfica na Engenharia e em outras ciências. Estas curvas usualmente são discretizadas como poligonais para serem manipuladas no computador. Para encontrar uma aproximação linear, ou discretização, de uma curva paramétrica basta discretizar o seu domínio. Este processo é conhecido como amostragem da curva onde podemos reconstruí-la simplesmente ligando as imagens dos pontos obtidos no domínio por segmentos de reta.

A diferença entre dois métodos de amostragem diferentes está nos pontos do domínio que são selecionados na sua discretização. Os melhores métodos são aqueles que resultam em poligonais que estão muito próximas à curva, com uma pequena quantidade de vértices. Estes dois objetivos são opostos, pois para melhorar uma aproximação geralmente é necessário adicionar novos pontos. Isto faz com que busquemos soluções que estabeleçam um compromisso entre os dois objetivos. 
O método de discretização mais popular é aquele que resulta da amostragem uniforme do domínio da curva. Embora muito simples, este método é altamente ineficiente na hora de aproximar a curva, pois depende fortemente do valor da derivada e pode acumular muitos pontos em algumas regiões, deixando outras descobertas. Como resultado, serão necessários muitos pontos para se obter uma boa aproximação para a curva. Pior ainda, é muito difícil (praticamente impossível) determinar a priori qual a quantidade de pontos satisfatória para uma boa aproximação da curva.

Métodos mais sofisticados procuram se adaptar à curvatura da curva como o método de Amostragem Adaptativa de Curvas Paramétricas (FIGUEIREDO, 1995) ou ao comprimento de arco. Os métodos baseados na curvatura amostram mais pontos onde a curva varia mais e dão uma boa aproximação da curva com poucos pontos. Os métodos baseados em comprimento de arco resultam em poligonais cujos lados têm aproximadamente o mesmo comprimento e ainda são (quase) proporcionais ao comprimento de arco da curva entre seus extremos. Estes podem não ser tão eficientes como os métodos baseados na curvatura, mas mesmo assim fornecem uma boa representação da curva e são muitos úteis em alguns problemas onde se deseja fazer uma interpolação ao longo da curva com velocidade constante, como é o caso da animação.

Neste artigo, estudaremos uma abordagem probabilística que é mais geral no sentido de que qualquer critério pode ser utilizado para a amostragem, incluindo os três citados acima. Cada critério é descrito por uma função de densidade definida no domínio da curva de onde amostraremos a variável aleatória correspondente. Esta amostragem satisfará o critério desejado, desde que a função de densidade tenha sido definida corretamente.

\section{Referencial teórico}

As seções 2 e 3 mostram a importância de todas as definições e teoremas que servirão de base para obter os resultados práticos apresentados no presente artigo.

\subsection{Definições}

Nesta subseção, as definições de variável aleatória contínua e função de densidade de probabilidade são os dois conceitos mais relevantes, uma vez que serão utilizados exaustivamente ao longo de todo o artigo. 
Definição 2.1. Chamaremos de experimento qualquer procedimento que pode ser realizado repetidas vezes, de maneira que, a cada novo ensaio, as condições de execução se mantenham inalteradas. Um experimento é aleatório se seus resultados puderem variar a cada ensaio, mesmo que as condições sejam as mesmas. Tais fenômenos precisam de um modelo matemático para seu estudo. São o que chamaremos de modelos não-determinísticos ou probabilísticos.

Definição 2.2. Sejam $\epsilon$ um experimento e $S$ um espaço amostral associado ao experimento. Uma função $X$, que associa a cada elemento $s \in S$ um número real, $X(s)$, é denominada variável aleatória.

O espaço $R_{x}$, conjunto de todos os valores possíveis de $\mathrm{X}$, é algumas vezes denominado contradomínio. De certo modo, poderemos considerar $R_{x}$ como um outro espaço amostral. O espaço amostral (original) $S$ corresponde ao resultado (possivelmente não-numérico) do experimento, enquanto $R_{x}$ é o espaço amostral associado à variável aleatória $X$, representando a característica que nos poderá interessar. Se for $X(s)=s$, teremos que $S=R_{x}$ (MEYER, 1983).

Definição 2.3. Sejam um experimento $\epsilon$ e seu espaço amostral $S$. Seja $X$ uma variável aleatória definida em $S$ e seja $R_{x}$ seu contradomínio. Seja $B$ um evento definido em relação a $R_{x}$, isto é, $B \subset R_{x}$. Então $A$ será definido assim:

$$
A=\{s \in S ; X(s) \in B\} .
$$

Neste caso, diremos que $A$ e $B$ são eventos equivalentes.

Definição 2.4. Sejam $\epsilon$ um experimento e seu espaço amostral $S$. A cada evento $A$ associaremos um número real representado representado por $P(A)$ e denominado probabilidade de $A$, que satisfaz às seguintes propriedades:

(1) $0 \leqslant P(A) \leqslant 1$;

(2) $P(S)=1$;

(3) Se $A$ e $B$ forem eventos mutuamente excludentes, $P(A \cup B)=P(A)+P(B)$;

(4) Se $A_{1}, A_{2}, \ldots, A_{n}, \ldots$ forem, dois a dois, eventos mutuamente excludentes, então,

$$
P\left(\bigcup_{i=1}^{\infty} A_{i}\right)=P\left(A_{1}\right)+P\left(A_{2}\right)+\ldots+P\left(A_{n}\right)+\ldots
$$

Definição 2.5. Seja $B$ um evento no contradomínio $R_{x}$. Neste caso, definimos $P(B)$ da seguinte maneira:

$$
P(B)=P(A)
$$

onde $A=\{s \in S ; X(s) \in B\}$. 
Definição 2.6. Se existir um número infinito de elementos no conjunto $A$, os quais possam ser postos em correspondência biunívoca com os inteiros positivos, diremos que $A$ é numerável ou infinito numerável.

Definição 2.7. Seja $X$ uma variável aleatória. Se o número de valores possíveis de $X$ (isto é, $R_{x}$, o contradomínio) for finito ou infinito numerável, denominaremos $X$ de variável aleatória discreta. Ou seja, os valores possíveis de $X$ podem ser postos em lista como $x_{1}, x_{2}, \ldots, x_{n}$. No caso finito, a lista acaba, e no caso infinito numerável, a lista continua indefinidamente.

Definição 2.8. Seja $X$ uma variável aleatória discreta. Portanto, $R_{x}$, o contradomínio de $X$, será formado no máximo por um número infinito numerável de valores $x_{1}, x_{2}, \ldots$ A cada possível resultado $x_{i}$ associaremos um número $p\left(x_{i}\right)=P\left(X=x_{i}\right)$, denominado probabilidade de $x_{i}$. Os números $p\left(x_{i}\right), i=1,2, \ldots$ devem satisfazer às seguintes condições:

$$
\begin{aligned}
& \text { (a) } \quad p\left(x_{i}\right) \geq 0, \forall i \\
& \text { (b) } \quad \sum_{i=1}^{\infty} p\left(x_{i}\right)=1 .
\end{aligned}
$$

A função $p$ definida acima, é denominada função de probabilidade (ou função de probabilidade no ponto) da variável aleatória $X$. A coleção de pares $\left[x_{i}, p\left(x_{i}\right)\right], i=1,2, \ldots$, é algumas vezes denominada distribuição de probabilidade de $X$.

Definição 2.9. Diz-se que $X$ é uma variável aleatória contínua, se existir uma função $f$, denominada de função de densidade de probabilidade (fdp) de $X$ que satisfaça às seguintes condições:

(a) $f(x) \geq 0$ para todo $x$;

(b) $\int_{-\infty}^{\infty} f(x) d x=1$;

(c) para quaisquer $a, b$, com $-\infty<a<b<\infty$, teremos $P(a \leq X \leq b)=\int_{a}^{b} f(x) d x$.

Definição 2.10. Seja $X$ uma variável aleatória, discreta ou contínua. Define-se a função $F$ como a função de distribuição acumulada da variável aleatória $X$ (indica-se por fd) como $F(x)=P(X \leq$ $x)$.

Lema 2.11. Se $A \subset B$, então $P(A) \leq P(B)$.

Prova. Podemos decompor $B$ em dois eventos mutuamentos excludentes, da seguinte forma: $B=$ $A \cup(B \cap \bar{A})$. Consequentemente, $P(B)=P(A)+P(B \cap \bar{A}) \geq P(A)$, porque $P(B \cap \bar{A}) \geq 0$, pela propriedade 1 da definição de probabilidade.

Teorema 2.12. Seja $X$ uma variável aleatória contínua com fdp $f$. Então,

(a) $F(x)=\int_{-\infty}^{x} f(s) d s$; 
(b) A função $F$ é não-decrescente, isto é, se $x_{1} \leq x_{2}$, teremos $F\left(x_{1}\right) \leq F\left(x_{2}\right)$;

(c) $f(x)=\frac{d}{d x} F(x)$, para todo $x$ no qual $F$ seja derivável.

Prova. (a) Decorre imediatamente da definição de variável aleatória contínua.

(b) Definamos os eventos $A$ e $B$, assim: $A=\left\{X \leq x_{1}\right\}, B=\left\{X \leq x_{2}\right\}$. Como $x_{1} \leq x_{2}$, teremos $A \subset B$, portanto, $P(A) \leq P(B)$ de acordo com o Lema 2.11 .

(c) $F(X)=P(X \leq x)=\int_{-\infty}^{x} f(s) d s$. Por isso, aplicando-se o teorema fundamental do cálculo, obteremos $F^{\prime}(x)=f(x)$.

Definição 2.13. Suponha-se que $X$ seja uma variável aleatória contínua, que tome todos os valores no intervalo $[a, b]$, no qual a e $b$ são ambos finitos. Se a fdp de $X$ for dada por

$$
f(x)=\left\{\begin{array}{ccc}
\frac{1}{b-a} & , & a \leq x \leq b ; \\
0 & , & x \notin[a, b] .
\end{array}\right.
$$

Diremos que $X$ é uniformemente distribuída sobre o intervalo $[a, b]$.

\section{Comentários:}

(a) Uma variável aleatória uniformemente distribuída tem uma fdp que é constante sobre o intervalo de definição. A fim de satisfazer à condição $\int_{-\infty}^{\infty} f(x) d x=1$, essa constante dever ser igual ao inverso do comprimento do intervalo.

(b) Para qualquer subintervalo $[c, d]$ em que $a \leq c<d \leq b, P(c \leq X \leq d)$ é a mesma para todos os subintervalos que tenham o mesmo comprimento. Isto é,

$$
P(c \leq x \leq d)=\int_{c}^{d} f(x) d x=\frac{d-c}{b-a}
$$

e, por isso, depende unicamente do comprimento do intervalo e não da posição desse intervalo.

\section{Metodologia}

Esta seção descreverá como será possível obter a quantidade de pontos aleatórios que amostrará uma curva plana a partir de qualquer função de densidade de probabilidade.

\subsection{Método da Transformação Inversa}

O método da Transformação Inversa será validado através da demonstração de um teorema. Tal método é na verdade o núcleo de toda a metodologia do trabalho. 
Suponhamos que temos uma distribuição probabilística, com função de densidade de probabilidade $f(x)$ e função de distribuição acumulada igual a $F(x)$. Desejamos gerar uma variável aleatória que siga essa distribuição probabilística. O método está baseado no fato de que a distribuição acumulada $F(x)$, tem valor entre 0 e 1 .

Teorema 3.1. Seja $X$ uma variável aleatória com fdp $f$ e $f d F$. [ Suponha que $f(x)=0, x \notin(a, b)$ ]. Seja $Y$ a variável aleatória definida por $Y=F(X)$. Então, $Y$ será uniformente distribuída sobre $[0,1]$. ( $Y$ é denominada transformada integral de $X$ ).

Prova. Primeiramente, provaremos que $F$ é uma função contínua. Tome uma sequência $x_{n}$ em $X(s)$ que convirja para $x$, ou seja, $x_{n} \rightarrow x$. Defina o evento $X \leq x_{n}$, portanto, $\left\{X \leq x_{n}\right\} \rightarrow\{X \leq x\}$. Logo, $P\left(X \leq x_{n}\right) \rightarrow P(X \leq x)$, então $F\left(x_{n}\right) \rightarrow F(x)$.

Logo, a função $F$ é contínua, estritamente crescente em $[a, b]$ e com uma inversa $F^{-1}$. Isto é, $Y=F(X)$ pode ser resolvida para $X$ em termos de $Y: X=F^{-1}(Y)$. [Como $F(x)=0$ para $x \leq a$, defina $F^{-1}(0)=a$. Semelhantemente, como $F(x)=1$ para $x \geq b$ defina $\left.F^{-1}(1)=b\right]$.

Seja $G$ a fd da variável aleatória $Y$, definida acima. Então,

$$
\begin{aligned}
G(Y) & =P(Y \leq y) \\
& =P(F(X) \leq y) \\
& =P\left(X \leq F^{-1}(y)\right) \\
& =F\left(F^{-1}(y)\right) \\
& =y .
\end{aligned}
$$

Consequentemente, a fdp de $Y$ é dada por $g(y)=G^{\prime}(y)=1$ para $0 \leq y \leq 1$. Portanto,

$$
f(x)=\left\{\begin{array}{cc}
1 & , \quad 0 \leq y \leq 1 \\
0, & x \notin[0,1] .
\end{array}\right.
$$

Logo, F é uniformemente distribuída sobre $[0,1]$.

Vamos, agora, dar alguns exemplos de geração de amostras aleatórias utilizando as distribuições uniforme e exponencial.

\subsection{Geração de amostras aleatórias}

Nesta seção mostraremos como gerar variáveis aleatórias a partir das distribuições uniforme e exponencial. 


\subsubsection{Distribuição Uniforme}

A função de distribuição acumulada da Distribuição Uniforme sobre o intervalo $[a, b]$ é dada por:

$$
f(x)=\int_{a}^{x} \frac{1}{b-a} d x=\frac{x-a}{b-a} .
$$

Seja:

$$
y=F(x)=\frac{x-a}{b-a} .
$$

Logo,

$$
x=a+(b-a) y
$$

A amostra aleatória é gerada, então, por:

$$
x_{i}=a+(b-a) y_{i}, i=1,2, \ldots, n
$$

onde $y_{1}, y_{2}, \ldots, y_{n}$ é uma sequência de números aleatórios uniformemente distribuídos em $[0,1]$.

\subsubsection{Distribuição Exponencial}

Definição 3.2. Uma variável aleatória contínua $X$, que tome todos os valores não-negativos, terá uma Distribuição Exponencial com parâmetro $\lambda>0$, se sua fdp for dada por

$$
f(x)=\left\{\begin{array}{ccc}
\lambda e^{-\lambda x} & , & x>0 \\
0 & , & \text { para quaisquer outros valores. }
\end{array}\right.
$$

A função de distribuição acumulada é dada por:

$$
F(x)=\int_{0}^{x} \lambda e^{-\lambda x} d x=1-e^{-\lambda x}
$$

Seja:

$$
y=F(x)=1-e^{-\lambda x}
$$

Logo,

$$
x=\frac{-\ln (1-y)}{\lambda} .
$$

A amostra aleatória é gerada, então, por:

$$
x_{i}=\frac{-\ln \left(1-y_{i}\right)}{\lambda}
$$

onde $y_{1}, y_{2}, \ldots, y_{n}$ é uma sequência de números aleatórios uniformemente distribuídos em $[0,1]$. 
Seja $X$ uma variável aleatória contínua e $f(x)$ uma função real. Se $f(x) \geq 0$ para todo $x$ e $\int_{-\infty}^{\infty} f(x) d x=K$, em que $K$ é um número real positivo (não necessariamente igual a 1), então $g(x)=\frac{f(x)}{K}$, é uma função de densidade de probabilidade, pois:

(i) $g(x) \geq 0$ para todo $x$;

(ii) $\int_{-\infty}^{\infty} g(x) d x=\int_{-\infty}^{\infty} \frac{f(x)}{K} d x=1$;

(iii) para quaisquer $a, b$, com $-\infty<a<b<\infty$, teremos $P(a \leq X \leq b)=\int_{a}^{b} g(x) d x$.

\subsubsection{Um pouco de Geometria Diferencial}

Vamos a partir de agora dar o conceito do que é uma curva plana e definir uma propriedade muito importante inerente a todas as curvas chamada de curvatura.

Definição 3.3. Uma curva parametrizada plana é uma aplicação diferenciável $\alpha: I \rightarrow R^{2}$ de um intervalo aberto $(a, b)$ da reta real $R$ em $\left.R^{2}\right)$.

Nesse caso, $\alpha$ é uma correspondência que leva cada $t \in I$ a um ponto da forma $\alpha(t)=$ $(x(t), y(t)) \in R^{2}$ onde $(x(t), y(t))$ são funções diferenciáveis (CARMO, 2005).

O vetor $\alpha^{\prime}(t)=\left(x^{\prime}(t), y^{\prime}(t)\right) \in R^{2}$ é chamado o vetor tangente da curva $\alpha$. A imagem $\alpha(I) \subset R^{2}$ é chamada de traço da curva.

Definição 3.4. Uma curva diferenciável parametrizada $\alpha: I \rightarrow R^{2}$ é chamada regular se $\alpha^{\prime}(t) \neq 0$ para todo $t \in I$.

Dado $t_{0} \in I$, o comprimento de arco de uma curva parametrizada regular $\alpha: I \rightarrow R^{2}$, a partir do ponto $t_{0}$, é por definição

$$
s(t)=\int_{t_{0}}^{t}\left|\alpha^{\prime}(t)\right| d t
$$

onde $\alpha^{t}(t)=\sqrt{\left(\left(x^{\prime}(t)\right)^{2}+\left(y^{\prime}(t)\right)^{2}\right)}$ é o comprimento do vetor $\alpha^{\prime}(t)$. Como $\alpha^{\prime}(t) \neq 0$, o comprimento de arco $s$ é uma função diferenciável de $t$ e $\frac{d s}{d t}=\left|\alpha^{\prime}(t)\right|$.

Definição 3.5. Seja $\alpha: I \rightarrow R^{2}$ uma curva parametrizada pelo comprimento de arco $s \in I$. $O$ número $\left\|\alpha^{\prime}(s)\right\|=k(s)$ chama-se curvatura de $\alpha$ em $s$.

Teorema 3.6. (DOMINGUES, 2013) Se uma curva dispor de uma parametrização $\alpha(t)=(x(t), y(t))$ não necessariamente pelo comprimento de arco, então será possível calcular a curvatura em cada t, através da expressão

$$
k(t)=\frac{\left|x^{\prime} y^{\prime \prime}-x^{\prime \prime} y^{\prime}\right|}{\left(\left(x^{\prime}\right)^{2}+\left(y^{\prime}\right)^{2}\right)^{\frac{3}{2}}} .
$$


Prova. Seja $s(t)$ a função comprimento de arco e denotemos sua inversa por $t(s)$. A reparametrização de $\alpha(t)$ pelo comprimento de arco é dada por:

$$
\beta(s)=\alpha(t(s))=(x(t(s)), y(t(s))) .
$$

Então,

$$
\beta^{\prime}(s)=\alpha^{\prime}(t(s)) \cdot t^{\prime}(s) .
$$

Daí,

$$
\begin{aligned}
\beta^{\prime \prime}(s) & =\alpha^{\prime \prime}(t(s)) \frac{d t}{d s} \frac{d t}{d s}+\alpha^{\prime}(t(s)) \frac{d}{d s}\left(\frac{d t}{d s}\right) \\
& =\alpha^{\prime \prime}(t(s))\left(\frac{d t}{d s}\right)^{2}+\alpha^{\prime}(t(s)) \frac{d^{2} t}{d s^{2}} .
\end{aligned}
$$

Como $\frac{d s}{d t}=\sqrt{\left(\left(x^{\prime}(t)\right)^{2}+\left(y^{\prime}(t)\right)^{2}\right)}$, então $\frac{d t}{d s}=\frac{1}{\sqrt{\left(\left(x^{\prime}(t)\right)^{2}+\left(y^{\prime}(t)\right)^{2}\right)}}$.

Daí,

$$
\frac{d^{2} t}{d s^{2}}=-\frac{\frac{1}{2} \cdot\left(\left(x^{\prime}\right)^{2}+\left(y^{\prime}\right)^{2}\right)^{-\frac{1}{2}} \cdot 2 \cdot\left(x^{\prime} x^{\prime \prime}+y^{\prime} y^{\prime \prime}\right)}{\left(\left(\left(x^{\prime}\right)^{2}+\left(y^{\prime}\right)^{2}\right)^{\frac{1}{2}}\right)^{2}}=-\frac{x^{\prime} x^{\prime \prime}+y^{\prime} y^{\prime \prime}}{\left(\left(x^{\prime}\right)^{2}+\left(y^{\prime}\right)^{2}\right)^{2}}
$$

Portanto,

$$
\begin{aligned}
k(s) & =\left\|\left(x^{\prime \prime}, y^{\prime \prime}\right)\left|\frac{1}{\left(x^{\prime}\right)^{2}+\left(y^{\prime}\right)^{2}}\right|+\left(x^{\prime}, y^{\prime}\right)\left(-\frac{x^{\prime} x^{\prime \prime}+y^{\prime} y^{\prime \prime}}{\left(\left(x^{\prime}\right)^{2}+\left(y^{\prime}\right)^{2}\right)^{2}}\right)\right\| \\
& =\left\|\left(\frac{x^{\prime \prime}\left(\left(x^{\prime}\right)^{2}+\left(y^{\prime}\right)^{2}\right)-x^{\prime}\left(x^{\prime} x^{\prime \prime}+y^{\prime} y^{\prime \prime}\right)}{\left(\left(x^{\prime}\right)^{2}+\left(y^{\prime}\right)^{2}\right)^{2}}, \frac{y^{\prime \prime}\left(\left(x^{\prime}\right)^{2}+\left(y^{\prime}\right)^{2}\right)-y^{\prime}\left(x^{\prime} x^{\prime \prime}+y^{\prime} y^{\prime \prime}\right)}{\left(\left(x^{\prime}\right)^{2}+\left(y^{\prime}\right)^{2}\right)^{2}}\right)\right\| \\
& =\left\|\left(\frac{x^{\prime \prime}\left(y^{\prime}\right)^{2}-x^{\prime} y^{\prime \prime} y^{\prime}}{\left(\left(x^{\prime}\right)^{2}+\left(y^{\prime}\right)^{2}\right)^{2}}, \frac{y^{\prime \prime}\left(x^{\prime}\right)^{2}-y^{\prime} x^{\prime \prime} x^{\prime}}{\left(\left(x^{\prime}\right)^{2}+\left(y^{\prime}\right)^{2}\right)^{2}}\right)\right\| \\
& =\left\|\frac{x^{\prime \prime} y^{\prime}-y^{\prime \prime} x^{\prime}}{\left(\left(x^{\prime}\right)^{2}+\left(y^{\prime}\right)^{2}\right)^{2}}\left(y^{\prime}, x^{\prime}\right)\right\| \\
& =\left|\frac{x^{\prime \prime} y^{\prime}-y^{\prime \prime} x^{\prime}}{\left(\left(x^{\prime}\right)^{2}+\left(y^{\prime}\right)^{2}\right)^{2}}\right|\left\|\left(y^{\prime}, x^{\prime}\right)\right\| \\
& =\left|\frac{x^{\prime \prime} y^{\prime}-y^{\prime \prime} x^{\prime}}{\left(\left(x^{\prime}\right)^{2}+\left(y^{\prime}\right)^{2}\right)^{2}}\right|\left(\left(x^{\prime}\right)^{2}+\left(y^{\prime}\right)^{2}\right)^{\frac{1}{2}} \\
& =\frac{\left|x^{\prime} y^{\prime \prime}-x^{\prime \prime} y^{\prime}\right|}{\left(\left(x^{\prime}\right)^{2}+\left(y^{\prime}\right)^{2}\right)^{\frac{3}{2}}} .
\end{aligned}
$$

\subsubsection{Amostragem aleatória com base na curvatura de uma curva plana}

Dada uma curva, podemos sempre definir uma função de densidade baseada na sua curvatura. Vejamos alguns exemplos. 
Exemplo 3.7. Vamos calcular a curvatura da curva plana parametrizada $r(t)=(t, \cosh (t)), t \in R$. Então,

$$
\begin{aligned}
k(t) & =\frac{|1 \cdot \cosh (t)-\sinh (t) \cdot 0|}{\left(1+\sinh ^{2}(t)\right)^{\frac{3}{2}}} \\
& =\frac{|\cosh (t)|}{\sqrt{\left(\cosh ^{2}(t)\right)^{3}}} \\
& =\frac{|\cosh (t)|}{\left|\cosh ^{3}(t)\right|} \\
& =\frac{1}{\cosh ^{2}(t)}>0 .
\end{aligned}
$$

Já vimos que $g(t)=\frac{k(t)}{K} d t$ onde $\int_{-\infty}^{\infty} k(t) d t=K$ é uma função de densidade de probabilidade.

Portanto,

$$
\begin{aligned}
g(t) & =\frac{\frac{1}{\cosh ^{2}(t)}}{\int_{a}^{b} \frac{1}{\cosh ^{2}(t)} d t} \\
& =\frac{\frac{1}{\cosh ^{2}(t)}}{\int_{a}^{b} \operatorname{sech}^{2}(t) d t} \\
& =\frac{\frac{1}{\cosh ^{2}(t)}}{\left.\tanh (t)\right|_{a} ^{b}} \\
& =\frac{\frac{1}{\cosh ^{2}(t)}}{\tanh (b)-\tanh (a)} \\
& =\frac{1}{\tanh (b)-\tanh (a)} \cdot \frac{1}{\cosh ^{2}(t)} .
\end{aligned}
$$

A função de distribuição acumulada $G(t)$ de $g(t)$ é dada por:

$$
\begin{aligned}
G(t) & =\frac{1}{\tanh (b)-\tanh (a)} \cdot \int_{a}^{t} \frac{1}{\cosh ^{2}(t)} d t \\
& =\frac{1}{\tanh (b)-\tanh (a)} \cdot(\tanh (t)-\tanh (a)) \\
& =\frac{\tanh (t)-\tanh (a)}{\tanh (b)-\tanh (a)} .
\end{aligned}
$$

Seja

$$
y=G(t)=\frac{\tanh (t)-\tanh (a)}{\tanh (b)-\tanh (a)}
$$


Logo,

$$
t=\tanh ^{-1}(y \cdot(\tanh (b)-\tanh (a))+\tanh (a)) .
$$

A amostra aleatória é gerada, então, por:

$$
t_{i}=\tanh ^{-1}\left(y_{i} \cdot(\tanh (b)-\tanh (a))+\tanh (a)\right), i=1,2, \ldots, n
$$

onde $y_{1}, y_{2}, \ldots, y_{n}$ é uma sequência de números aleatórios uniformemente distribuídos em [0,1].

Exemplo 3.8. A circunferência tem parametrização $r(t)=(r \cdot \cos (t), r \cdot \sin (t))$ onde $r$ é o raio da circunferência e $t \in R$. Sua curvatura é constante, pois

$$
\begin{aligned}
k(t) & =\frac{|r \cdot \sin (t) \cdot r \cdot \sin (t)+r \cdot \cos (t) \cdot r \cdot \cos (t)|}{\left((r \cdot \sin (t))^{2}+(r \cdot \cos (t))^{2}\right)^{\frac{3}{2}}} \\
& =\frac{\left|r^{2}\left(\sin ^{2}(t)+\cos ^{2}(t)\right)\right|}{\left(r^{2}\left(\sin ^{2}(t)+\cos ^{2}(t)\right)\right)^{\frac{3}{2}}} \\
& =\frac{r^{2}}{\sqrt{r^{6}}} \\
& =\frac{1}{r}>0 .
\end{aligned}
$$

Então, $g(t)=\frac{\frac{1}{r}}{\int_{a}^{b} \frac{1}{r} d t}=\frac{1}{\int_{a}^{b} d t}=\frac{1}{b-a}$ é uma função de densidade de probabilidade.

A função de distribuição acumulada é dada por:

$$
G(t)=\int_{a}^{t} \frac{1}{b-a} d t=\frac{t-a}{b-a} .
$$

Seja:

$$
y=G(t)=\frac{t-a}{b-a}
$$

Logo, $t=a+(b-a) y$.

A amostra aleatória é gerada, então, por:

$$
t_{i}=a+(b-a) y_{i}, i=1,2, . ., n
$$

onde $y_{1}, y_{2}, \ldots, y_{n}$ é uma sequência de números aleatórios uniformemente distribuídos em $[0,1]$.

\subsubsection{Descrição do algoritmo}

Nesta seção daremos uma descrição do algoritmo utilizado para gerar a amostragem aleatória de pontos. 
O primeiro passo é definir uma função de densidade de probabilidade $f(x)$ definida no domínio da curva que se deseja aproximar. Em seguida, calcula-se a função de distribuição acumulada $F(x)$.

Faça $y=F(x)$ e depois coloque a variável independente $x$ em função de $y$. Gere uma sequência de números aleatórios uniformemente distribuída no intervalo $[0,1]$. Calcule $x_{i}, i=1,2, \ldots, n$ para cada $y_{i}$ gerado.

\section{Resultados e discussão}

Todas as curvas paramétricas geradas neste trabalho utilizaram uma amostragem aleatória de 200 pontos uniformemente distribuída no intervalo [0,1]. É importante frisar que qualquer quantidade de pontos poderia ser utilizada desde que tal quantidade gere uma boa aproximação da curva. Outro fato que merece destaque, é que o trabalho em questão não fornece um método para determinar um valor mínimo de pontos que aproxime de forma satisfatória uma curva plana parametrizada. A quantidade de pontos é encontrada através de tentativa e erro. Nesta seção, os pontos em azul representam a amostra aleatória no domínio da curva plana parametrizada e os pontos em vermelho são os valores da curva sobre a parte do domínio discretizado (amostra aleatória).

\subsection{Aproximação de curvas utilizando a Distribuição Uniforme}

Figura $1-\alpha(t)=\left(t^{3}, t^{2}\right)$. Intervalo de aproximação: $[-100,100]$.

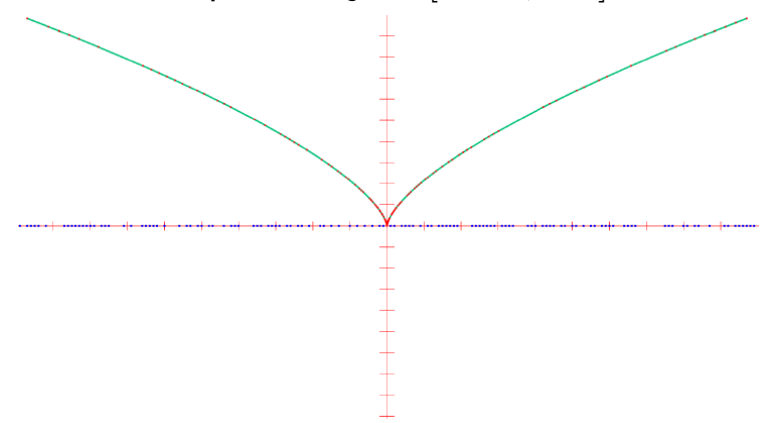

Fonte: Elaboração dos autores (2020).
Figura 2- $\alpha(t)=\left(t, t^{2}\right)$. Intervalo de aproximação: $[-100,100]$.

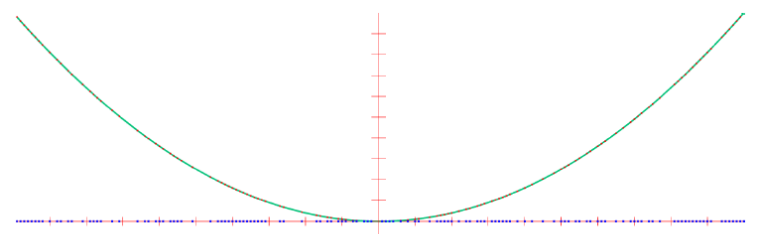

Fonte: Elaboração dos autores (2020). 
Figura $3-\alpha(t)=\left(t, t^{3}\right)$. Intervalo de aproximação: $[-100,100]$.

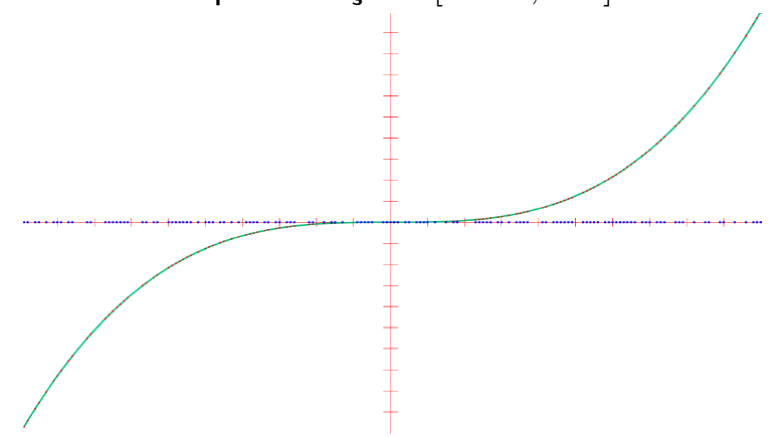

Fonte: Elaboração dos autores (2020).
Figura $4-\alpha(t)=(t, \cos (t))$. Intervalo de aproximação: $[-360,360]$.

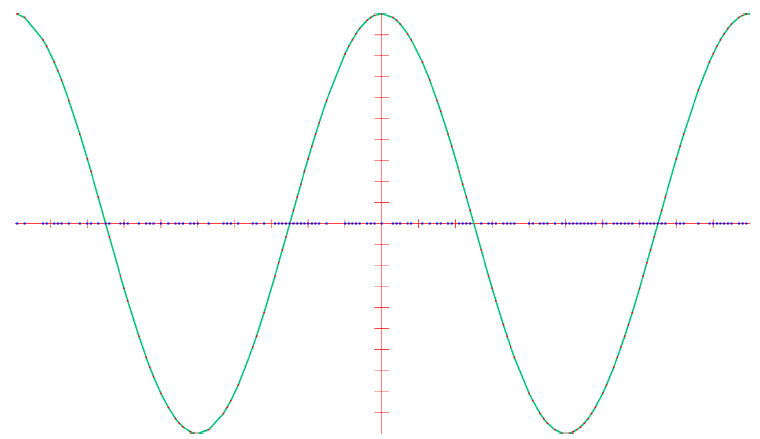

Fonte: Elaboração dos autores (2020).

Figura $5-\alpha(t)=(\cos (t), \sin (2 t))$. Intervalo de aproximação: $[0,360]$.

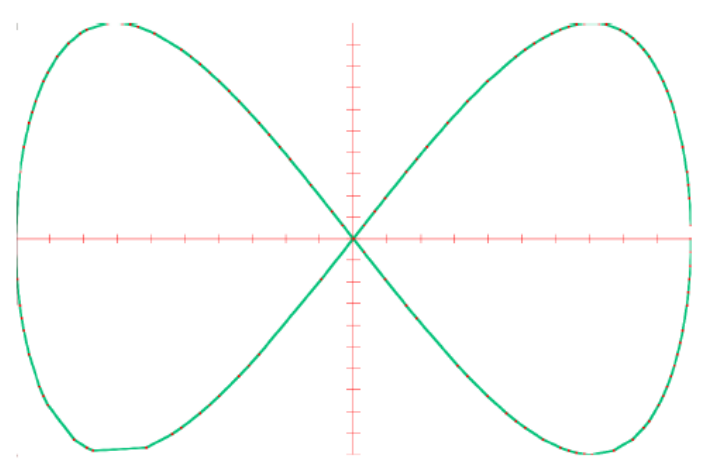

Fonte: Elaboração dos autores (2020).

Figura $6-\alpha(t)=(t, \cosh (t))$. Intervalo de aproximação: $[-180,180]$.

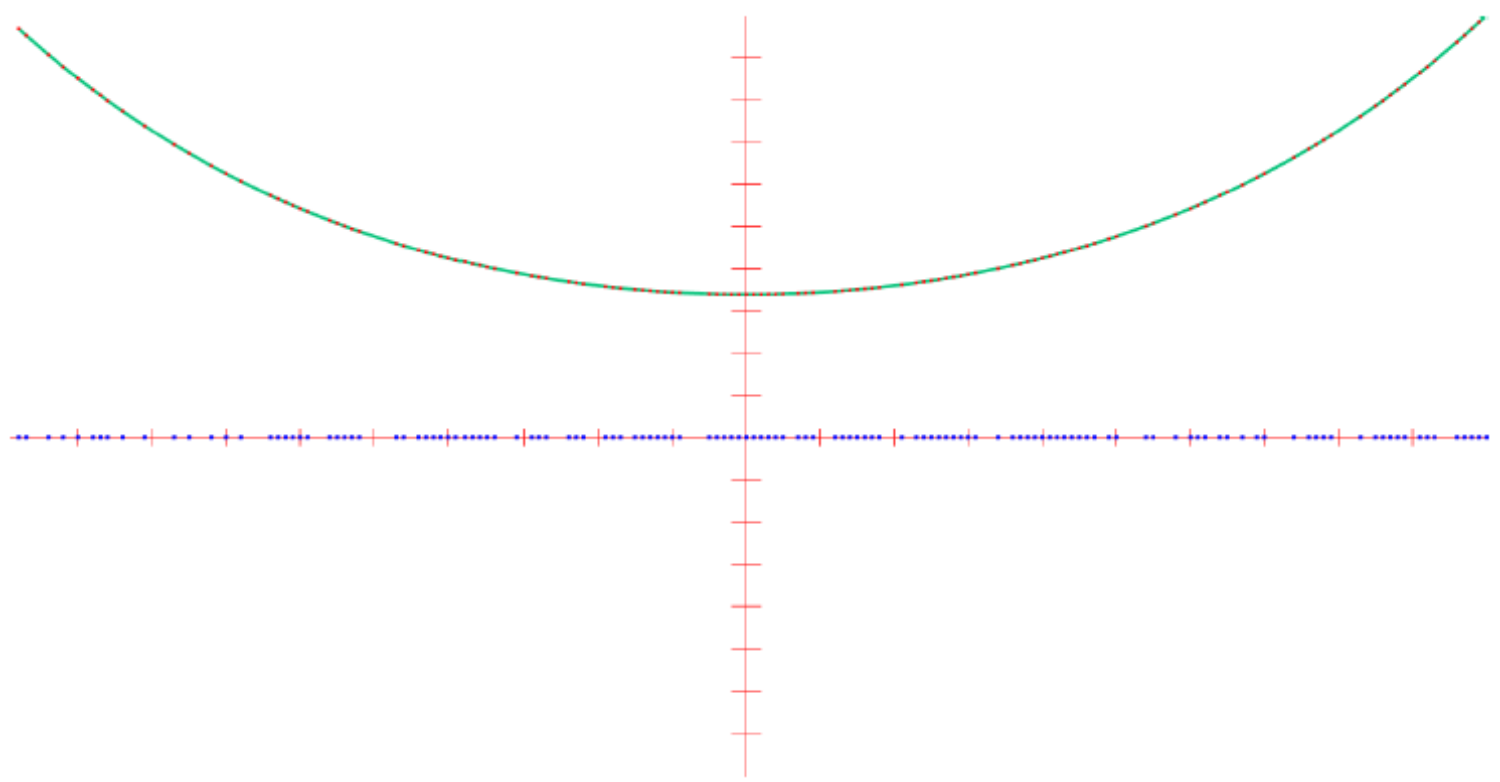

Fonte: Elaboração dos autores (2020). 
Foram utilizados outros intervalos e outras amostragens com quantidade de pontos diferentes para aproximar as curvas das Figuras 1, 2, 3, 4, 5 e 6. Em todos os casos, chegou-se a uma boa aproximação do modelo contínuo da curva. Note que houve uma boa distribuição dos pontos ao longo do eixo $x$, ou seja, foram amostrados tantos pontos negativos como positivos. A única exceção foi o gráfico da Figura 5, onde só foram amostrados pontos positivos uma vez que os pontos estão no intervalo $[0,360]$. Porém, como a curva é simétrica em relação ao eixo $y$, foi possível aproximá-la de forma satisfatória. A escolha dos intervalos de aproximação depende da eurística na qual nem sempre um intervalo de amplitude maior com mais pontos amostrados significa uma melhora visível na aproximação da curva.

\subsection{Aproximação de curvas utilizando a Distribuição Exponencial}

Figura $7-\alpha(t)=\left(t^{3}, t^{2}\right)$. Intervalo de aproximação: $[-100,100]$.

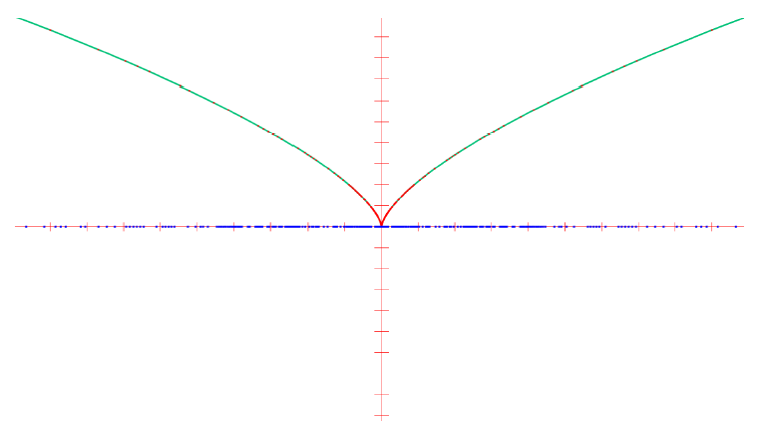

Fonte: Elaboração dos autores (2020).

Figura $9-\alpha(t)=\left(t, t^{3}\right)$. Intervalo de aproximação: $[-100,100]$.

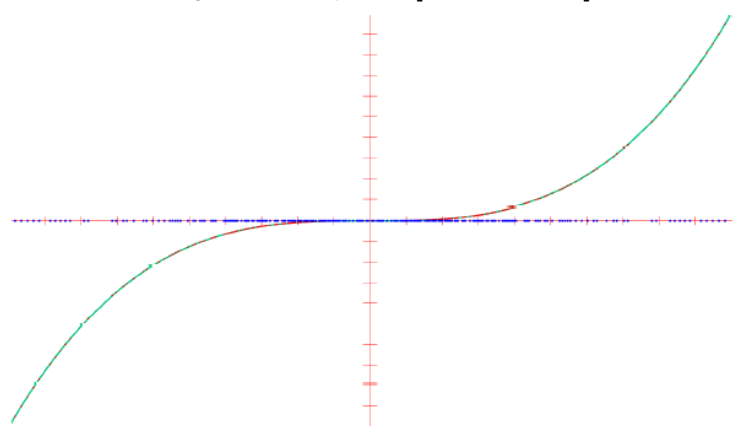

Fonte: Elaboração dos autores (2020).
Figura $8-\alpha(t)=\left(t, t^{2}\right)$ Intervalo de aproximação: $[-100,100]$.

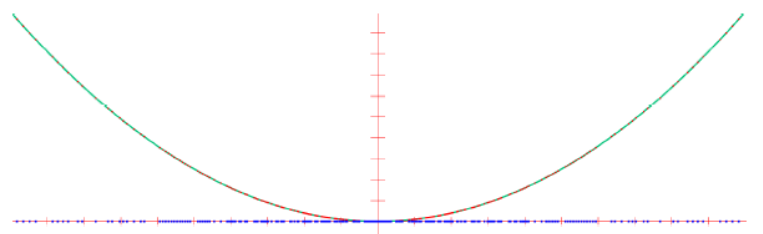

Fonte: Elaboração dos autores (2020).

Figura $10-\alpha(t)=(t, \cos (t))$. Intervalo de aproximação: $[-360,360]$.

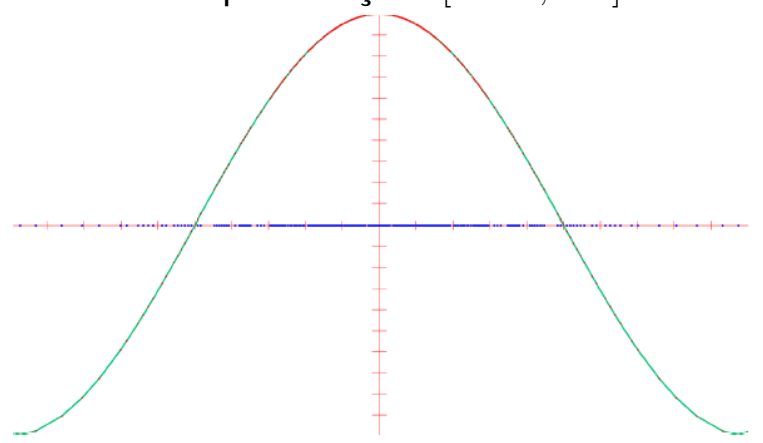

Fonte: Elaboração dos autores (2020). 
Figura $11-\alpha(t)=(t, \cosh (t))$. Intervalo de aproximação: $[-180,180]$.

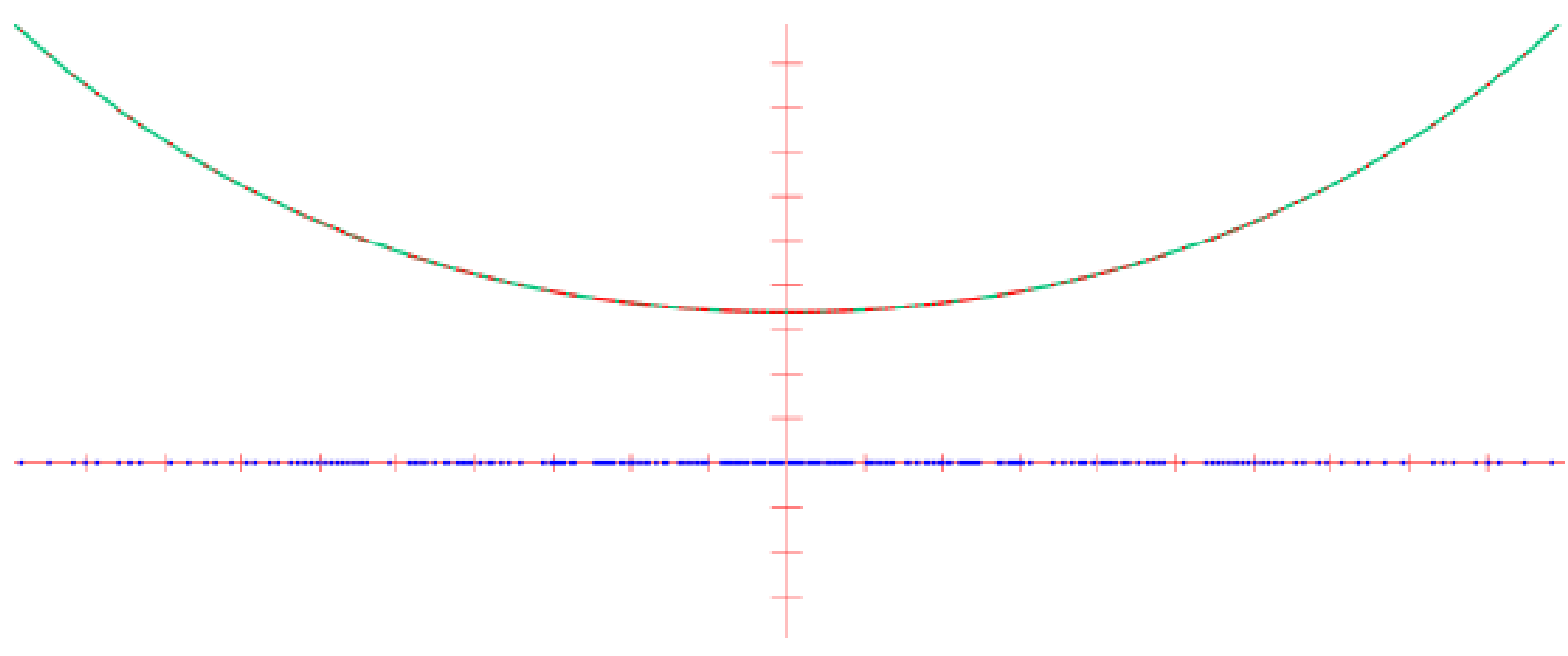

Fonte: Elaboração dos autores (2020).

Foram utilizados outros intervalos, vários valores da constante $\lambda$ e amostragens com quantidade diferentes de pontos distribuídos uniformemente no intervalo $[0,1]$ para aproximar as curvas das Figuras 7, 8, 9, 10 e 11, porém tais mudanças não resultaram em mudanças significativas nas aproximações. Como foi dito anteriormente, o valor de $\lambda$ não influenciou na aproximação das curvas, mas é importante frisar que tal valor, geralmente, é bem pequeno e se for um número maior do que 1 pode interferir negativamente na aproximação das curvas.

Figura $12-\alpha(t)=(\cos (t), \sin (2 t))$. Intervalo de aproximação: $[0,360]$ e $\lambda=0,03$.

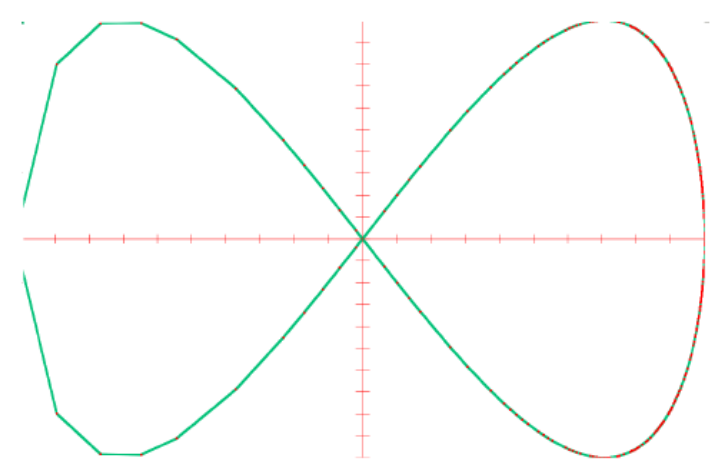

Fonte: Elaboração dos autores (2020). 
Figura $13-\alpha(t)=(\cos (t), \sin (t))$. Intervalo de aproximação: $[0,360]$ e $\lambda=0,02$.

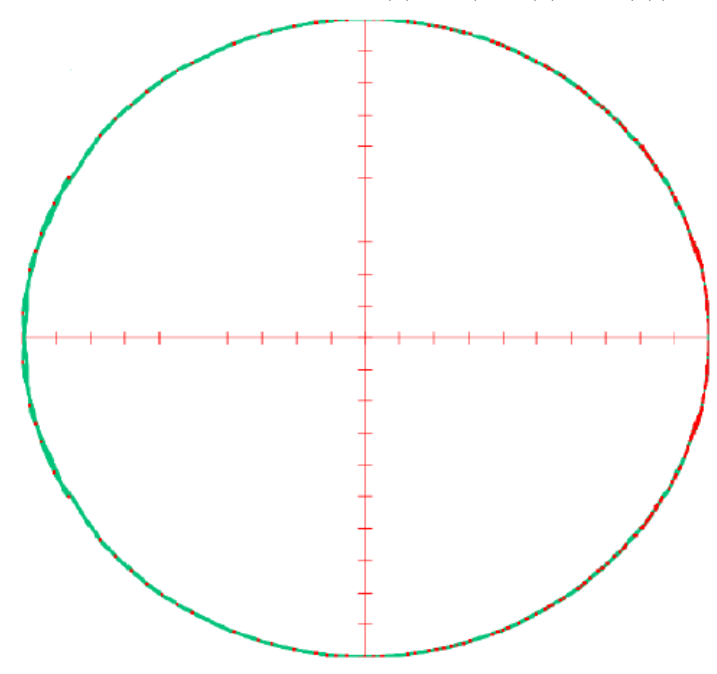

Fonte: Elaboração dos autores (2020).

Foram utilizados outros intervalos, vários valores da constante $\lambda$ e amostragens com quantidade diferentes de pontos distribuídos uniformemente no intervalo $[0,1]$ para aproximar as curvas das Figuras 12 e 13, porém o valor da constante $\lambda$ foi o único fator que interferiu na aproximação da curva sendo, portanto, necessário encontrar através da heurística um valor adequado.

\subsection{Aproximação de curvas com base na curvatura}

Figura $14-\alpha(t)=(\cos (t), \sin (t))$. Intervalo de aproximação: $[0,360]$.

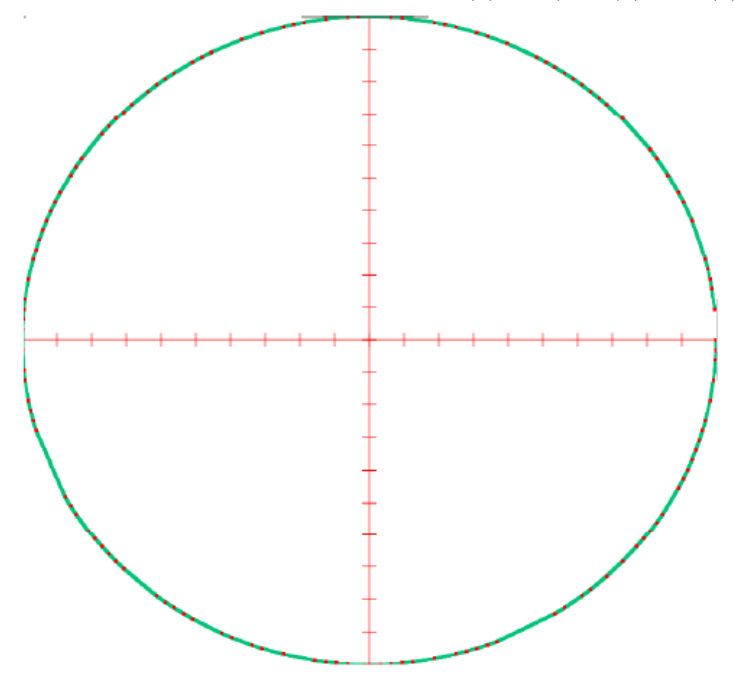

Fonte: Elaboração dos autores (2020). 
Figura $15-\alpha(t)=(t, \cosh (t))$. Intervalo de aproximação: $[-180,180]$.

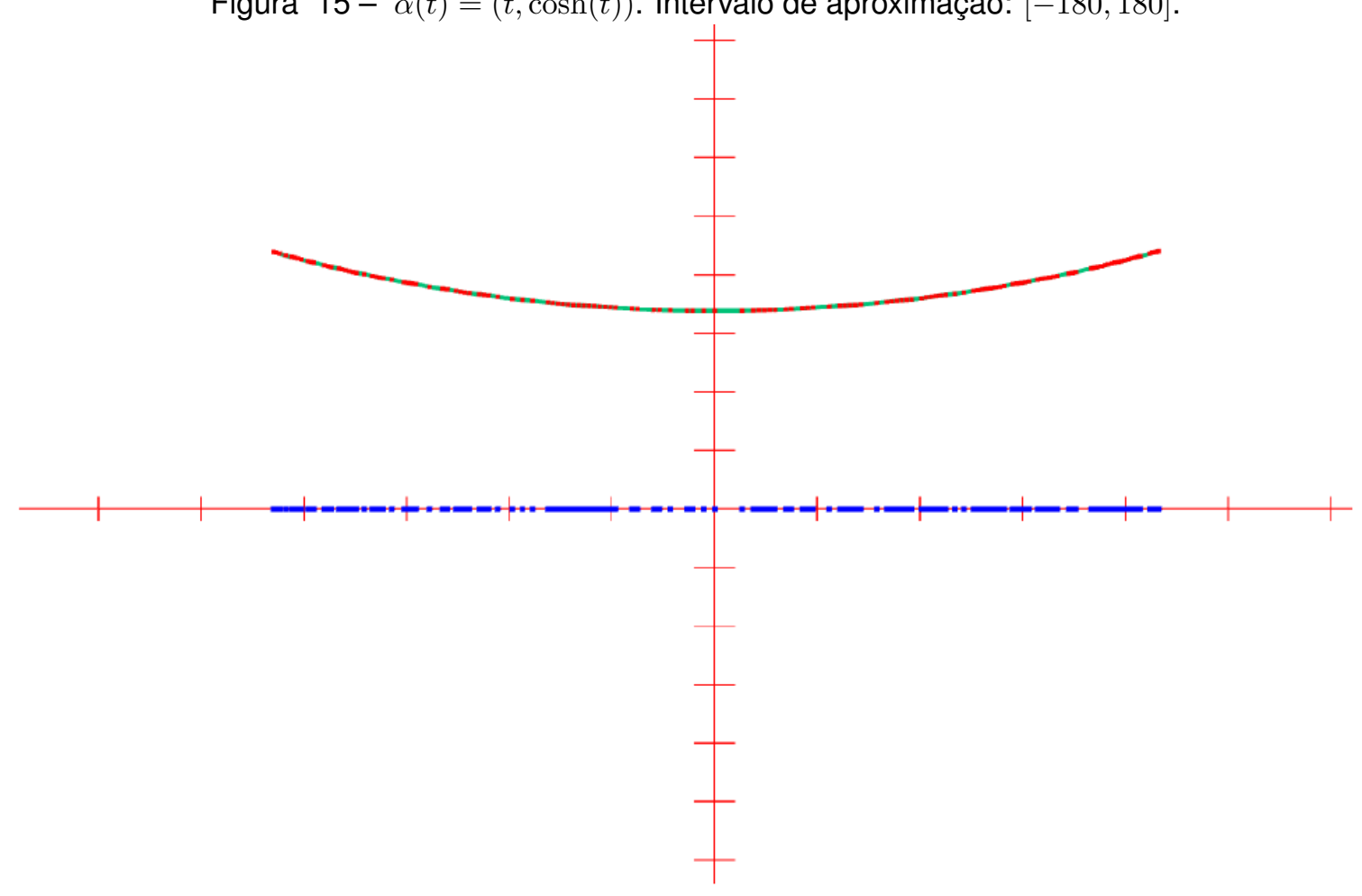

Fonte: Elaboração dos autores (2020).

A curvatura da circunferência da Figura 14 é constante e por isso, verificou-se que a amostragem de pontos ficou mais ou menos uniforme no domínio da curva. A amostragem de pontos no domínio da curva da Figura 15 se concentrou onde a curvatura é maior. A principal dificuldade em aproximar uma curva com base na sua curvatura é integrar a função curvatura que nem sempre é uma tarefa fácil. Uma alternativa para superar tal dificuldade pode ser realizar uma integração aproximada através de algum método de integração numérica.

\section{Considerações finais}

Em geral, aproximar curvas planas com base na Distribuição Uniforme se revelou mais eficiente do que tomando como referência a Distribuição Exponencial. $O$ valor de $\lambda$ na Distribuição Exponencial interfere na aproximação de algumas curvas, sendo necessário encontrar um valor de $\lambda$ adequado para chegar a uma boa aproximação da curva.

Vale ressaltar, que poderia ser utilizada qualquer Função de Densidade de Probabilidade definida no domínio da curva. Este trabalho, apenas mostra como encontrar adequadamente uma amostragem de pontos que irá aproximar a curva. 
Por último, falamos sobre os resultados obtidos com a amostragem de pontos no domínio da curva baseada na curvatura. Aproximar a curva com base na sua curvatura é um método usado quando se deseja gerar mais amostras onde a curvatura é maior. Porém, esse método só pode ser usado em casos especiais uma vez que a integral da função curvatura, na maioria das vezes, é muito difícil de ser calculada.

\section{Referências Bibliográficas}

CARMO, M. P. do. Geometria Diferencial de Curvas e Superfícies. 3. ed. Rio de Janeiro: SBM, 2005.

DOMINGUES, J. P. F. Geometria Diferencial das Curvas Planas. 2013. 71 f. Orientador: Thiago de Melo. Dissertação (Mestrado Profissional em Matemática) - Universidade Estadual Paulista Júlio de Mesquita Filho, Instituto de Geociências e Ciências Exatas, Rio Claro, 2013.

FIGUEIREDO, L. H. de. Adaptive Sampling of Parametric Curves. In: PAETH, A. W. (Org.). Graphics Gems V. Cambridge: Academic Press, 1995. p. 173-178.

MEYER, P. L. Probabilidade: Aplicações à Estatística. Trad. FILHO, R. C. B. L. 2. ed. Rio de Janeiro: LTC, 1983. 